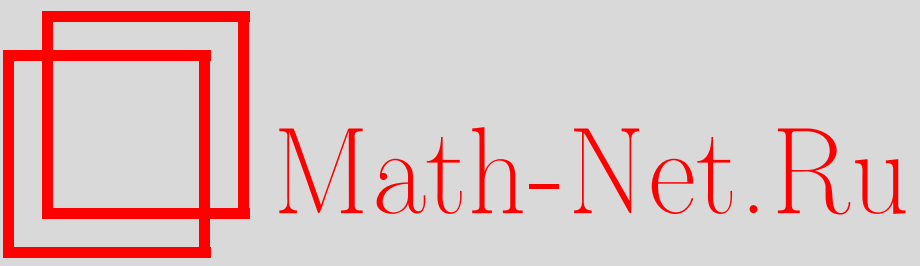

А. В. Романов, О предельной динамике эволюционных уравнений, УМН, 1996, том 51, выпуск 2, 173-174

DOI: https://doi.org/10.4213/rm962

Использование Общероссийского математического портала Math-Net.Ru подразумевает, что вы прочитали и согласны с пользовательским соглашением

http://www.mathnet.ru/rus/agreement

Параметры загрузки:

IP : 54.198 .187 .58

26 апреля 2023 г., $17: 02: 37$ 


\title{
О ПРЕДЕЛЬНОЙ ДИНАМИКЕ ЭВОЛЮЦИОННЫХ УРАВНЕНИЙ
}

\author{
A. В. Романов
}

Предлагается каноническое преобразование полулинейных уравнений параболического типа, позволяющее во многих случаях построить для данного уравнения инерциальное многообразие и соответствующую инерииальную форму - ОДУ в $\mathbb{R}^{k}$ с аналогичной динамикой при большом времени. Этот подход дает возможность значительно расширить список асимптотически конечномерных параболических задач, что и демонстрируется на примере систем реакции-диффузии.

1. Пусть $X$ - сепарабельное гильбертово пространство и

$$
\dot{u}=-A u+F(u)
$$

есть полулинейное параболическое диссипативное уравнение в $X$. Здесь $u=u(t) \in X, t \geqslant 0$, $A$ - линейный самосопряженный положительный оператор с компактной резольвентой, гладкая нелинейность $F$ в известном смысле "подчинена" $A$ и $\|u(t)\|_{X}<\rho=$ const для $t>t^{*}(u(0))$. Конечномерное (диффеоморфное области $U \subset \mathbb{R}^{k}$ ) инвариантное и экспоненциально притягивающее все решения $u(t)$ гладкое многообразие $M \subset X$ называем инерииальныцм многообразием уравнения (1). Сужение (1) на $M$ индуцирует инерциальную форм⿻ - ОДУ в $U$, полностью описывающее поведение $u(t)$ при $t \rightarrow+\infty$.

2. Рассмотрим в области $\Omega \subseteq \mathbb{R}^{N}$ систему реакции-диффузии

$$
u_{t}=D \Delta u+f(u)
$$

с однородными условиями Дирихле, Неймана или с условиями периодичности на $\partial \Omega$. Здесь $\Omega-$ область с гладкой границей или параллелепипед, $u(t, x)=\left(u_{1}, \ldots, u_{n}\right), D=d I$ - скалярная числовая $(n \times n)$-матрица с $d>0$ и нелинейная функция $f \in C_{0}^{3}\left(\mathbb{R}^{n} ; \mathbb{R}^{n}\right)$ (что, в частности, обеспечивает диссипативность $(2)$ в $\left.L^{2}\left(\Omega ; \mathbb{R}^{n}\right)\right)$. Пусть $\mu_{1} \leqslant \mu_{2} \leqslant \cdots-$ спектр $-\Delta_{\Omega}$, где $\Delta_{\Omega}-$ оператор Лапласа в $\Omega$ с одним из данных краевых условий.

Известно [1], что система (2) допускает инерциальное многообразие, если $\sup \left(\mu_{s+1}-\mu_{s}\right)$ $=\infty$ по $s \geqslant 1$; последнее справедливо для $\Omega=(0, a)$, но не всегда верно уже для $\Omega \subseteq \mathbb{R}^{2}$. В [2] установлено существование инерциального многообразия у скалярного $(n=1)$ уравнения $(2)$ в произвольном прямоугольнике $\Omega \subset \mathbb{R}^{2}$, кубе $\Omega \subset \mathbb{R}^{3}$ и (на уровне анонсирования) в параллелепипеде $\Omega \subset \mathbb{R}^{3}$ с рационально соизмеримыми квадратами ребер.

3. На основе изложенной в п. 4 общей схемы построения инерциальных многообразий и инерциальных форм уравнений вида (1) получены следующие приложения к системам (2).

Теорема 1. Система (2) допускает инерциальное многообразие в $L^{2}\left(\Omega ; \mathbb{R}^{n}\right)$ для случаев:

а) $\Omega$ - прямоугольник или ограниченная область в $\mathbb{R}^{2}$ с гладкой границей;

б) $\Omega$ - параллелепипед в $\mathbb{R}^{3}$ с рационально соизмеримыми квадратами ребер.

4. Специфическая техника [3], [4] позволяет сводить (в определенном понимании) некоторые нелинейные уравнения параболического типа к ОДУ в $\mathbb{R}^{k}$. Здесь предлагается стандартная процедура подобного рода для абстрактного уравнения (1). Положим

$$
v=-A^{-1} F(u), \quad J(u)=(u, v(u)) .
$$

Если $u(t)$ - решение $(1)$, то $\nu(t)=(u(t), v(t))$ - решение эволюционного уравнения

$$
\dot{\nu}=-B \nu+G_{0}(\nu)
$$


с фазовым пространством $E=X \times X$, линейным оператором $B(u, v)=(A u+A v, A v)$ и нелинейностью

$$
G_{0}(u, v)=(0, g(u)), \quad g(u)=-F(u)+A^{-1} F^{\prime}(u)(A u-F(u)),
$$

где $F^{\prime}$ - производная Фреше и $F(u)=A^{-1} A F(u)$ при $F(u) \in$ области определения $A$. Подходящая замена $G_{0}$ на нелинейность $G$ с $G(\nu) \equiv G_{0}(\nu)$ "в окрестности" множества $J(K)(K$ - глобальный аттрактор (1)) приводит к диссипативному в $E$ эволюционному уравнению

$$
\dot{\nu}=-B \nu+G(\nu)
$$

Пусть $T=\operatorname{Re} B, T(u, v)=(A u+A v / 2, A u / 2+A v)-$ положительный оператор в $E$ и $\left\{E^{\theta}, T\right\}$ - шкала гильбертовых пространств с нормой $\|\nu\|_{\theta}=\left\|T^{\theta} \nu\right\|_{E}$. Спектры $\sigma(\cdot)$ операторов $T$ и $A$ связаны так: $2 \sigma(T)=\sigma(A) \cup 3 \sigma(A)$. Допустим, что $G$ - гладкое равномерно-липшищево отображение $E^{\alpha}$ в $E^{\beta}$ (при некоторых $\alpha, \beta \geqslant 0, \alpha-\beta<1$ ). Методика [5] позволяет тогда установить существование инерциального многообразия $M \subset E^{\alpha} \subset E$ уравнения (4) в предположении

$$
\sup _{k \geqslant 1}\left(\lambda_{k+1}-\lambda_{k}\right) /\left(\lambda_{k+1}^{\alpha-\beta}+\lambda_{k}^{\alpha-\beta}\right)=\infty
$$

где $\left\{\lambda_{1} \leqslant \lambda_{2} \leqslant \cdots\right\}=\sigma(T)$ и, возможно, $\alpha-\beta<0$. Оказывается, в ряде случаев условие (5) выполняется для уравнения (4), хотя и не выполнено для (1) (с гильбертовой шкалой $\left\{X^{\theta}, A\right\}$ ). Наконец, в данной ситуации удается показать, что наличие у (4) инерциального многообразия в $E$ влечет за собой существование инерциального многообразия исходного уравнения (1) в $X$. Дальше эта схема применяется к системе (2) в частном случае $n=1, N=2$ с условием Дирихле на $\partial \Omega$ и $f(0)=0$.

5. Запишем (2) в виде (1) с $X=L^{2}(\Omega)$ и $A=-d \Delta_{\Omega}$. Преобразования (3) дают

$$
A g(u)=d f^{\prime \prime}(u)|\nabla u|^{2}-f^{\prime}(u) f(u)
$$

Можно заменить (как указано выше) $G_{0}$ на нелинейность $G$, обеспечивающую диссипативность в (4) и равномерно-липшицеву из $E^{\alpha}$ в $E^{\beta}$ с $\alpha \in(3 / 4,1), \beta=1$. Важно, что здесь $\alpha<\beta$, тогда как нелинейная часть (2) равномерно-липшицева в шкале $\left\{X^{\theta}, A\right\}$ с $\alpha, \beta=0$. Пользуясь свойствами $\sigma\left(\Delta_{\Omega}\right)$ для областей $\Omega \subseteq \mathbb{R}^{2}$ и связью $\sigma(T)$ с $\sigma(A)$, находим, что спектр $\sigma(T)$ удовлетворяет (5) при $\alpha<\beta$. Таким образом, уравнение (4) допускает инерциальное многообразие, откуда выводим и существование инерциального многообразия для уравнения (2).

6. Итерируя преобразования (3), можно расширить границы применимости теоремы 1 , а также доказать наличие инерциального многообразия у двумерной системы Навье-Стокса на торе с произвольными периодами, что сушественно усиливает резултаты [3].

\section{СПИСОК ЛИТЕРАТУРЫ}

[1] Камаев Д.А. // Записки научн. семин. ЛОМИ АН СССР. 1981. Т. 110. С. 57-73. [2] Mallet-Paret J., Sell G.R. // J. Amer. Math. Soc. 1988. V. 1. №4. P. 805-866. [3] Kwak M. // Indiana Univ. Math. J. 1992. V. 41. № 4. P. 927-981. [4] Baek J., Ju H.-K., Kwak M. // Indiana Univ. Math. J. 1993. V. 42. №3. P. 721-731. [5] Романов А. В. // Изв. РАН. Сер. матем. 1993. Т. 57. № 4. С. 36-54. 